\title{
Correction to: Anesthesia for Intense Pulsed Light (IPL) Treatments
}

Ileana Mitre and Calin Mitre

\section{Correction to: L. Fodor, Y. Ullmann (eds.), Aesthetic Applications of Intense Pulsed Light, https://doi.org/10.1007/978-3-030-22829-3_5}

The original version of this chapter was revised.

In section 5.3.1.4, the dosage was inadvertently published and now it has been updated as $300 \mathrm{mg} /$ administration. 